José Carval ho de Noronha 1,2

\title{
Avaliação da relação entre volume de procedimentos e a qualidade do cuidado: o caso de cirurgia coronariana no Brasil
}

\author{
Volume and quality of care \\ in coronary artery bypass grafting in Brazil
}

Claudia Travassos 2

Mônica Martins 3

Mônica R. Campos 4

Paula Maia 2

Rogério Panezzuti 2

\footnotetext{
1 Instituto de Medicina Social, Universidade do Estado do Rio de Janeiro. Rua São Francisco Xavier 524 7 o andar, Rio de Janeiro, RJ 20550-900, Brasil. noronha@uerj.br 2 Departamento de Informações em Saúde, Centro de Informação Científica e Tecnológica, Fundação Oswaldo Cruz. Avenida Brasil 4365, Rio de Janeiro, RJ 21045-900, Brasil. 3 Departamento de Administração e Planejamento em Saúde,

Escola Nacional de Saúde Pública, Fundação Oswaldo Cruz. Rua Leopoldo Bulhões 1480, Rio de Janeiro, RJ 21042-210, Brasil.

4 Departamento de Ciências Sociais, Escola Nacional de Saúde Pública, Fundação Oswaldo Cruz. Rua Leopoldo Bulhões 1480, Rio de Janeiro, RJ 21042-210, Brasil.
}

Abstract There is often a negative association between the volume of medical procedures and hospital mortality rates. The objective of this study is to determine whether this association is observed in Brazilian hospitals performing coronary artery bypass grafts (CABG) that were financed by the Ministry of Health. CABG surgeries performed from 1996 to 1998 were analyzed. Data were collected from the Ministry of Health Hospital Information System. The statistical procedure utilized was the Cox survival analysis. Hospitals were grouped in classes of volume of CABG performed. The model was adjusted to the severity of cases (risk of dying). From January 1996 to December 1998 the M inistry of Heal th rei mbursed 41,989 CABG procedures in 131 hospitals. The overall hospital mortality rate was 7.2\%. A gradient was observed in the mortality rates. The severity-adjusted risk of dying in the group of hospitals performing more procedures was lower than in the group performing fewer operations. It is recommended that the Ministry of Health strongly consi der regionalized concentration of services to perform CABG.

Key words Surgery; Health Services; Hospitalization; Hospital Mortality

Resumo O volume de procedi mentos médi cos geral mente apresenta-se negativamente associado com a taxa de mortalidade hospitalar. O objetivo deste trabal ho é verificar nos hospi tais brasileiros a existência ou não dessa associação no caso das cirurgias de revascularização do mi ocárdio (CRVM), financiadas pelo Ministério da Saúde (MS). Analisaram-se as CRVM realizadas de 1996 a 1998. Os dados foram obti dos por intermédio do Si stema de Informações Hospital ares do SUS. O procedimento estatístico utilizado foi análise de sobrevida (model o de Cox). Os hospitais foram agrupados em classes de volume de CRVM. O model o foi ajustado pel o perfil de gravidade (risco de morrer) dos pacientes. Foram pagas 41.989 CRVM pelo MS entrejanei ro de 1996 e dezembro de 1998, realizadas em 131 hospitais. A taxa de mortalidade foi de 7,2\%. Observou-se um gradiente crescente nas taxas à medida que diminuiu o volume. No grupo de hospitais do SUS com maior volume de CRVM os pacientes operados apresentaram menor risco de morrer do que no de hospitais com menor volume de cirurgias. Recomenda-se que o SUS deva estimular a concentração regional izada dos servi ços para a realização de CRVM.

Palavras-chave Cirurgia; Serviços de Saúde; Hospitalização; Mortalidade Hospitalar 


\section{Intro dução}

A avaliação da qualidade dos serviços de saúde encontra nos trabal hos de Donabedian (1980) seu grande marco referencial. Já se tornou clássica a divisão das abordagens para essa avaliação em termos de estrutura, processo e resultado. Atualmente, existe um interesse crescente pelas medidas de resultado. Como salienta Palmer (1991), as medidas baseadas em resultados são particularmente interessantes porque tratam da finalidade última do cuidado em saúde, que é a melhoria da saúde dos pacientes.

A taxa mortalidade hospitalar tem sido freqüentemente utilizada como indicador de resultado em estudos sobre o desempenho hospitalar (Travassos et al., 1999). Pesquisas apontam grande variabilidade desse indicador entre hospitais, mas indicam resultados contraditórios sobre as causas dessa variação. Estudos sobre determinantes dos problemas na qualidade do cuidado nos hospitais, que utilizam as taxas de mortalidade como indicador de qualidade, apontam para a existência de diversos fatores explicativos (Fink et al., 1989; Luft et al., 1990; OTA, 1988; Thomas \& Hofer, 1998; Travassos et al., 1999). Alguns desses indicam que o grau de organização e de comunicação do corpo clínico, a experiência e a especialização dos profissionais aparecem associados à melhoria de qualidade (Dubois et al., 1987; Kelly \& Hellinger, 1987; OTA, 1988). Com relação à natureza jurídica do serviço, observou-se um padrão de cuidado mel hor nos hospitais não lucrativos. Os hospitais com maior estabilidade financei ra também tiveram um desempenho meIhor (OTA, 1988).

O volume de procedimentos realizados tem sido apresentado como um fator negativamente associado com a taxa de mortalidade. Isto é, o volume geralmente mostra-se positivamente correlacionado com a qualidade do cuidado (Birkmeyer et al., 2002; Luft et al., 1990; SpiegeIhater, 2002; Wennberg et al., 1987).

No Brasil, estudou-se pouco a relação entre volume e qualidade do cuidado hospitalar (J. C. Noronha, em memória apresentada à Academia Nacional de Medicina) $O$ objetivo deste trabal ho é verificar nos hospitais brasileiros a existência ou não dessa associação. Mais particularmente examinar a existência de relação entre o volume e as taxas de mortalidade nas cirurgias de revascularização do miocárdio (CRVM), financiadas pelo Ministério da Saúde (MS). A taxa de mortalidade hospitalar após cirurgia coronariana é um indicador do desempenho do cuidado prestado, indicador de resultado largamente utilizado na avaliação da qualidade deste procedimento cirúrgico nos Estados Unidos (Hannan et al., 1991; Luft et al., 1990). O resultado da cirurgia depende da qualidade do atendimento oferecido e de fatores de risco do paciente, prévios à cirurgia (Tu et al., 1997).

\section{Materiais e métodos}

Analisaram-se as CRVM pagas pelo Sistema Ú nico de Saúde (SUS) nos anos de 1996 a 1998. Essas correspondem a uma parcela das CRVM realizadas no país, pois não incluem aquelas financiadas por Planos Privados de Saúde ou pagas do próprio bolso. Os dados foram obtidos por intermédio do Sistema de Informações Hospitalares do SUS (SIH-SUS). As internações hospitalares pagas com recursos do MS são processadas para efeitos de pagamento pelo $\mathrm{SIH}$ SUS, implantado em 1991 (MS, 2000). Cada internação é classificada em um procedimento realizado com base na Tabela de Procedimentos (DATASUS, 1999), el aborada para efeito de pagamento. Procedimento Realizado é a unidade de pagamento do mecanismo de reembolso aos hospitais adotado pelo SUS. Os dados são divulgados pelo DATASUS em CD-ROM para cada um dos municípios do país com periodicidade mensal. Esses dados podem ser divididos em seis blocos: identificação do hospital; identificação do paciente; identificação do médico responsável pelo paciente; caracterização da internação; campo de procedimentos especiais e campo de serviços profissionais.

Os dados disponíveis no SIH-SUS são agrupados em diferentes arquivos, obrigando no caso desta pesquisa o uso de procedimento de integração de dados entre arquivos para a geração do banco de dados da pesquisa. Tendo em vista a complexidade do processo de integração de dados, desenvolveu-se uma estratégia voltada para aumentar a sua eficiência e diminuir as chances de erro. $O$ banco de dados da pesquisa resultou da integração de três arquivos abaixo destacados e o processo de integração de dados está descrito em detalhes em artigo publicado anteriormente (Campos et al., 2000):

- Arquivos Movimento de AlH (tipo MA), cujos registros correspondem a cada AlH enviada pelos hospitais, agregadas por município e por mês.

- Arquivos Movimento de Prestadores (tipo MT), cujos registros correspondem a cada ato médico realizado em cada internação, por prestador direto de serviço, agregados pelo país e por mês. Os registros contêm a variável (Número da AlH) que identifica cada internação. 
- Arquivos Procedimentos Autorizados na AlH (tipo PA), cujos registros correspondentes a cada Procedimento Especial realizado em cada internação, agregados por município e por mês.

Selecionaram-se apenas os registros com Procedimento Realizado "Cirurgia Coronariana com Extracorpórea” - código 32011016. É importante ressaltar que os casos de CRVM realizada sem a utilização de circulação extracorpórea também eram codificados como tal, pela ausência de codificação específica no período estudado, constituindo uma fonte de heterogeneidade entre os casos. Dado o caráter agudo da intervenção analisada, a problemática de dupla contagem de casos por inclusão indevida de registros de pacientes crônicos e fora de possibilidade terapêutica (AlH tipo 5) não se verifica. Os hospitais são identificados pelo seu número de inscrição no Cadastro Geral de Contribuintes (CGC).

No estudo da relação entre volume e resultado do cuidado as transformações matemáticas e as categorias de volume influenciam os achados dos estudos (Luft et al., 1990). Assim, após testar diferentes formas de categorização da variável volume, optou-se por trabalhar essa variável agrupada em cinco classes de volume deste procedimento (<150 cirurgias, 151 a 250 cirurgias, 251 a 450, 451 a 600 e mais do que 600 cirurgias).

Em função da distribuição assimétrica dos óbitos em relação ao tempo de permanência do paciente no hospital (Figura 1), utilizou-se a regressão de Cox (Cox proportional-harzards models) (Rothman \& Greenland, 1998), que permite o teste da associação entre volume de cirurgias e óbito controlado pelo tempo que o paciente permaneceu no hospital. Variações no tempo médio de permanência entre hospitais decorrentes de diferenças nas práticas de admissão e de alta, no acesso diferenciado ao cuidado domiciliar e a serviços de reabilitação, podem influenciar o local de ocorrência do óbito e conseqüentemente as taxas de mortalidade hospitalar (OTA, 1988). Por outro lado, a diferença no tempo de permanência pode refletir, parcialmente, as diferenças na gravidade dos pacientes, mas podem também ser resultado de cuidado inefetivo e de uma maior incidência de complicações intra-hospitalares (Jencks et al., 1988). A variável "tempo de permanência no hospital" assumiu o valor 0,5 dia para os pacientes que "saíram" do hospital na mesma data da internação. O modelo foi ajustado pelo perfil de gravidade (risco de morrer) dos pacientes submetidos à cirurgia em cada um dos hospitais estudados. A internação com realização de CRVM foi a unidade de análise e o volume foi medido em nível do hospital. As variáveis de gravidade selecionadas foram aquelas que se mostraram associadas ao risco de morrer em pacientes submetidos a CRVM, em estudo anterior que utilizou os dados do $\mathrm{SIH}$ SUS (Noronha, 2001). O fato de utilizar-se da-

Figura 1

Distribuição do tempo de permanência das internações (AlH) por cirurgias de revascularização do miocárdio (CRVM), segundo o resultado do cuidado: alta e óbito. Brasil, 1996-1998.

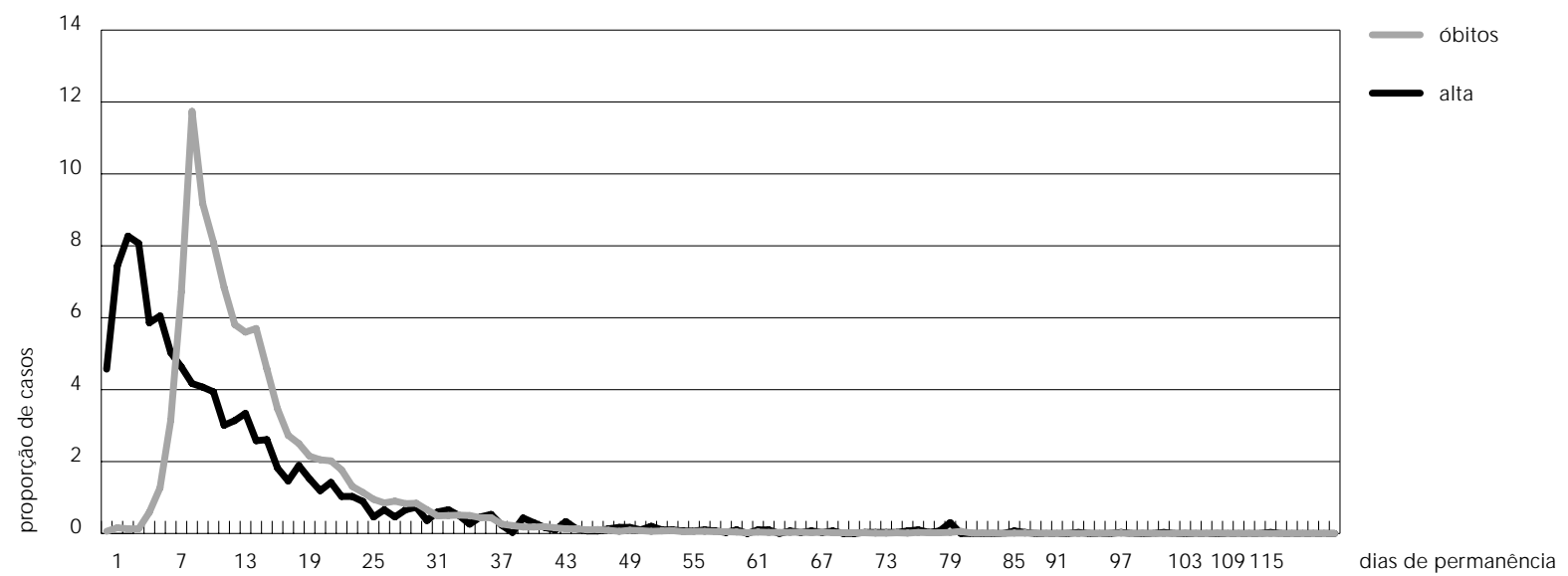


dos originários de banco de dados administrativos representa uma limitação deste estudo, na medida em que esse banco contém um número limitado de informações sobre os fatores de risco de morrer dos pacientes.

O modelo testado incluiu como controle da gravidade do paciente os seguintes fatores de risco: idade, sexo, uso dos procedimentos especiais - "albumina" e "nutrição parenteral" - e dias de permanência do paciente na Unidade de Terapia Intensiva (UTI). O uso de procedimentos especiais e o tempo de permanência na UTI foram utilizados como medidas indiretas (variáveis proxy) das condições clínicas dos pacientes. A idade foi tratada em três categorias: até 40 anos, de 40 a 59 anos e mais de 60 anos, com a faixa inicial como categoria de referência. O sexo masculino assumiu a função de categoria de referência. Criou-se a variável dicotômica "procedimento especial" representando a realização ou não dos procedimentos "albumina" e/ ou "nutrição parenteral". Foram criadas as seguintes faixas de dias de permanência do paciente na UTI: até 3 dias (categoria de referência), de 3 a 9 e mais que 9 dias. Utilizaram-se os pontos de corte que expressavam nítidas áreas de padrões diferenciados de associação com o óbito, que refletiam as mudanças de padrão nas curvas. Por exemplo, no caso da variável "dias de permanência do paciente na UTI", do terceiro ao nono dia a curva dos pacientes que faleceram mostrou-se abaixo da curva dos pacientes que sobreviveram, havendo uma inversão a partir deste valor. Para volume de cirurgias, a categoria de referência foi a classe de volume maior que 600 cirurgias. O programa utilizado para análise dos dados foi o SPSS versão 10.0 para sistema operacional Windows.

\section{Resultados}

No Brasil, foram pagas 41.989 CRVM pelo MS entre janeiro de 1996 e dezembro de 1998. A taxa de mortalidade foi de 7,2 óbitos hospitalares por 100 cirurgias (Tabela 1). A idade média dos pacientes foi de 59,9 anos e $35,4 \%$ das cirurgias foram realizadas em pacientes com idade acima de 64 anos. O sexo masculino representou $67,5 \%$ dos casos. Em média os pacientes permaneceram 15 dias hospitalizados. O uso de albumina e nutrição parenteral ocorreu em $24 \%$ dos casos. Os pacientes permaneceram em média 3,8 dias na UTI (Tabela 1 ).

A taxa de mortalidade hospitalar variou para as diferentes classes de volume de cirurgias (Tabela 2). Essas cirurgias foram realizadas em
131 hospitais, localizados em 22 unidades da federação. A maior parte das cirurgias foi realizada em hospitais que realizaram ao menos 251 procedimentos nos anos de estudo. Contudo, o número de hospitais que realizaram até 150 cirurgias é grande (55 unidades hospitalares). Observou-se um gradiente crescente nas taxas à medida que diminuiu o volume. A taxa de mortalidade hospitalar dos pacientes operados nos hospitais que realizaram mais de 600 cirurgias nos três anos foi a menor $(5,81 \%)$ e essa aumentou progressivamente à medida que o volume de cirurgias decresceu.

A Tabela 3 apresenta o modelo final utilizado para testar a relação entre volume e mortalidade hospitalar. No modelo inicial testado, a variável "procedimento especial" não se mostrou estatisticamente associada ao risco de morrer. As pessoas com 60 anos ou mais apresentaram o maior risco de morrer após CRVM (RR = 1,72 ) e as pessoas com idade entre 40 e 59 anos apresentaram um risco morrer comparativamente menor do que as mais idosas, entretanto o coeficiente nesta faixa etária não foi significativo, sua permanência no modelo final justifica-se pela importância da idade como fator de confundimento na análise do efeito da classe de volume. O risco de morrer foi maior entre as mulheres ( $R R=1,35)$; as pessoas com permanência na UTI de duração muito alta (>9 dias) apresentaram maior risco de morrer do que aquelas com permanência de duração média ( 3 a 9 dias), cujo risco relativo foi 0,68 .

Com o emprego da regressão de Cox rejeitou-se a hipótese nula de ausência de efeito do volume de cirurgias sobre a mortalidade hospitalar após a CRVM (Tabela 3). Ceteris paribus, observou-se uma associação entre volume e resultado da cirurgia que mostrou um "efeito dose-resposta". Os resultados do teste foram estatisticamente significativos ( $p<0,000$ ) para as quatro classes de volume estudadas (Tabela 3). Com relação aos hospitais que realizaram mais de 600 cirurgias no período estudado, os que realizaram até 150 cirurgias e os hospitais que realizaram entre 151 e 250, apresentaram riscos relativos superiores, respectivamente de 2,15 e 1,88 .

\section{Discussão}

Este estudo mostrou que no grupo de hospitais do SUS com maior volume de CRVM os pacientes operados apresentaram menor risco de morrer do que no grupo de hospitais com menor volume de cirurgias. Este resultado está em concordância com os de grande parte dos 
Tabela 1

Características da população de estudo.

\begin{tabular}{|c|c|}
\hline \multicolumn{2}{|l|}{ Características da população } \\
\hline \multicolumn{2}{|l|}{ Número de casos no período de estudo } \\
\hline 1996 & 13.680 casos \\
\hline 1997 & 13.897 casos \\
\hline 1998 & 14.412 casos \\
\hline Total no período (1996-1998) & 41.989 casos \\
\hline \multicolumn{2}{|l|}{ Demográficas } \\
\hline Idade média & 59,9 anos $( \pm 10,1)$ \\
\hline Mediana & 61 anos \\
\hline Moda & 63 anos \\
\hline \multicolumn{2}{|l|}{ Faixa etária } \\
\hline$<40$ anos & 1.038 casos $(2,5 \%)$ \\
\hline $40-59$ anos & 18.131 casos $(43,2 \%)$ \\
\hline$>59$ anos & 22.820 casos $(54,3 \%)$ \\
\hline Idosos (> 64 anos) & 14.880 casos $(35,4 \%)$ \\
\hline Sexo (homem) & 28.360 casos $(67,5 \%)$ \\
\hline \multicolumn{2}{|l|}{ Permanência no hospital } \\
\hline 0-7 dias & 6.257 casos $(14,9 \%)$ \\
\hline 8-14 dias & 21.313 casos $(50,9 \%)$ \\
\hline $15-29$ dias & 11.483 casos $(27,3 \%)$ \\
\hline $30-400$ dias & $2.876 \operatorname{casos}(6,8 \%)$ \\
\hline Média & 14,5 dias $( \pm 11)$ \\
\hline Mediana & 12 dias \\
\hline Moda & 8 dias \\
\hline \multicolumn{2}{|l|}{ Permanência na UTI } \\
\hline $0-2$ dias & 19.274 casos $(45,9 \%)$ \\
\hline 3-9 dias & 20.185 casos $(48,1 \%)$ \\
\hline 9 dias & $2.530 \operatorname{casos}(6,0 \%)$ \\
\hline Média & 3,8 dias $( \pm 4)$ \\
\hline Mediana & 3 dias \\
\hline Moda & 2 dias \\
\hline \multicolumn{2}{|l|}{ Procedimento especial } \\
\hline Uso de albumina e nutrição parenteral & 10.065 casos $(24,0 \%)$ \\
\hline \multicolumn{2}{|l|}{ Resultado do cuidado } \\
\hline Óbitos & $3.023 \operatorname{casos}(7,2 \%)$ \\
\hline
\end{tabular}


Taxas de mortalidade hospitalar após cirurgia de revascularização do miocárdio (CRVM), não ajustadas por hospitais agrupados por classes de volume de cirurgias. Brasil, 1996-1998.

\begin{tabular}{lrcc}
\hline Classes de volume & Casos (\%) & Hospitais & $\begin{array}{c}\text { Taxa de mortalidade } \\
\text { hospitalar não ajustada (\%) }\end{array}$ \\
\hline$>600$ & $19.029(45,3)$ & 13 & 5,81 \\
$451-600$ & $5.985(14,3)$ & 12 & 7,17 \\
$251-450$ & $8.203(19,5)$ & 23 & 8,53 \\
$151-250$ & $5.465(13,0)$ & 28 & 8,98 \\
$\leq 150$ & $3.307(7,9)$ & 55 & 9,00 \\
\hline
\end{tabular}

Tabela 3

Modelo de regressão de Cox para testar a relação entre volume e resultado (óbito) das cirurgias de revascularização do miocárdio (CRVM).

\begin{tabular}{|c|c|c|c|}
\hline Variáveis & Coeficiente & Erro-padrão & Risco relativo (IC 95\%) \\
\hline Sexo feminino & $0,297^{*}$ & 0,037 & $1,345(1,251-1,447)$ \\
\hline \multicolumn{4}{|l|}{ Idadel } \\
\hline 40-59 anos & $0,137^{* *}$ & 0,145 & $1,146(0,863-1,523)$ \\
\hline$>60$ anos & $0,541^{*}$ & 0,143 & $1,719(1,298-2,276)$ \\
\hline \multicolumn{4}{|l|}{ Dias na UTI2 } \\
\hline 3-9 dias & $-0,381^{*}$ & 0,041 & $0,683(0,631-0,741)$ \\
\hline$>9$ dias & $0,374^{*}$ & 0,050 & $1,454(1,319-1,602)$ \\
\hline \multicolumn{4}{|l|}{ Volume de casos $^{3}$} \\
\hline$<151$ cirurgias & $0,763^{*}$ & 0,066 & $2,145(1,885-2,441)$ \\
\hline $151-250$ cirurgias & $0,633^{*}$ & 0,055 & $1,882(1,691-2,095)$ \\
\hline $251-450$ cirurgias & $0,561^{*}$ & 0,049 & $1,752(1,593-1,927)$ \\
\hline $451-600$ cirurgias & $0,540^{*}$ & 0,057 & $1,716(1,533-1,920)$ \\
\hline
\end{tabular}

1 Categoria de referência: idade $<39$ anos.

2 Categoria de referência: dias de permanência na UTI: < 3 dias.

3 Categoria de referência: volume de casos: $>600$ cirurgias.

$*$ valor $=0,000$

** $\mathrm{p}$ valor $=0,346$.

estudos internacionais sobre volume e mortalidade hospitalar, em particular no caso da CRVM. Birkmeyer et al. (2002), que encontraram uma associação moderada entre volume e mortalidade hospitalar no caso da CRVM, acreditam que a consistência nos resultados dos estudos já realizados indica a existência de uma associação causal entre volume do procedimento e qualidade do cuidado hospitalar. Sobretudo para hospitais que realizam menos de 150 procedimentos por ano.

Shroyer et al. (1996), utilizando dados da rede de 43 hospitais dos Veteranos dos Estados Unidos não puderam confirmar as relações entre volume e mortalidade hospital ar quando as taxas foram ajustadas. Entretanto, Clark (1996), utilizando a base de dados nacional da Sociedade de Cirurgia Torácica estadunidense, examinando 124.793 cirurgias realizadas por mais de 1.200 cirurgiões em mais de 600 hospitais, observou que aqueles hospitais que operaram menos de 100 casos por ano apresentaram taxa de mortalidade superior (5,0\%) à esperada (3,2\%). Tu \& Naylor (1996), confirmaram esta associação em hospitais canadenses em Ontário.

A relação entre volume e mortalidade hospitalar pós CRVM já foi analisada com a utiliza- 
ção de bases de dados administrativos (Luft et al., 1987) · Hannan et al. (1989) publicaram estudo pioneiro utilizando as bases administrativas do Estado de Nova I orque. Neste trabaIho foi observado que hospitais que realizavam mais de 223 cirurgias anuais (valor mediano para o Estado), apresentavam taxas de mortalidade significativamente inferiores aos hospitais de menor volume (risco relativo de 0,74 ). Em estudo posterior, utilizando a casuística de três anos, Hannan et al. (1991) confirmaram o "efeito protetor" para os hospitais de alto volume (ponto de corte de 200 cirurgias, risco relativo de 0,84$)$, com considerável variação entre os hospitais de baixo volume.

As principais críticas a esses estudos dizem respeito centralmente às metodologias de ajuste, particularmente quando se utilizam bases de dados administrativos. $\mathrm{O}$ ajuste insuficiente da gravidade dos casos neste estudo pode ter resultado em erro na estimativa do efeito de volume. Entretanto, estudo anterior (Noronha, 2001), que testou o ajuste por gravidade em pacientes submetidos a CRVM comparando a precisão de modelos de predição do risco de morrer que utilizam as variáveis disponíveis no SIH-SUS com modelos que incluem variáveis clínicas coletadas nos prontuários médicos, mostrou que apesar de suas limitações, as bases de dados de informações hospitalares permitem, por meio do exame das variáveis demográficas, de saídas e de procedimentos especiais, pelo menos analisar o desempenho comparado dos vários hospitais para aquelas condições em que a mortalidade hospitalar seja um evento de relevância.

Sowden et al. (1995) reviram 15 estudos publicados entre 1972 e 1992 que examinaram as relações entre volume de CRVM e mortalidade hospitalar, examinando-os em relação ao grau de ajustamento de risco dos casos estudados, concluindo que aqueles estudos com ajustes de perfis de casos mais adequados apresentavam razões de chance mais próximas de um, reduzindo as estimativas dos benefícios do alto volume. Entretanto, estes achados não foram confirmados por outros autores. Tu el al. (1997) procuraram examinar se modelos mais abrangentes para ajuste de riscos poderiam ter um impacto mais significativo nas taxas de mortalidade ajustadas por risco após cirurgia de revascularização do miocárdio na província de Ontário, no Canadá. Esses autores compararam o impacto de modelos mais abrangentes versus aqueles limitados a fatores de risco cruciais. Para isso, efetuaram estudo avaliando impacto incremental de modelos de ajuste de risco crescentemente mais abrangente utili- zando os fatores de risco cirúrgico. Os resultados apontam que uma grande quantidade de informações prognósticas pode ser obtida com relativamente poucas variáveis clínicas.

U ma outra limitação do presente estudo é o fato de ter-se trabalhado apenas com as internações financiadas pelos SUS. Em hospitais que atendem pacientes do SUS e pacientes financiados com recursos privados o volume de cirurgias realizadas foi subestimado. Infelizmente, os dados disponíveis não permitem uma estimativa do volume de cirurgias com financiamento privado por hospital. Deve-se também analisar com cautela as estimativas de erro padrão, pois na modelagem estatística empregada não se corrigiu para o fato de ter-se trabalhado com variável de âmbito do hospital no modelo de indivíduos. Entretanto, o efeito de volume permanece estatisticamente significativo mesmo quando se aumenta o intervalo de confiança para 99,9\%.

Outro aspecto a ser consi derado, é que a associação aqui observada entre volume e resultado não permite inferência sobre cada hospital isoladamente. Isto por que este estudo não avaliou o efeito independente de volume sobre o resultado da cirurgia. O volume de procedimentos embora manifeste associação com resultados, não é o único ou principal fator responsável por taxas de mortalidade hospitalar mais elevada. Outras características dos hospitais e dos prestadores diretos de cuidados, que não foram consideradas neste estudo, também podem explicar a qualidade do cuidado.

Luft et al. (1990) já haviam demonstrado que diferentes agrupamentos de volume levam a resultados distintos, enfatizando que os benefícios de volume se evidenciam quando confrontados com grupos de baixo volume, menos de 100 casos.

A revisão feita pelo Centro de Economia da Saúde da Universidade de York (NHS Centre for Reviews \& Dissemination, 1997) para vários procedimentos cirúrgicos, inclusive a CRVM, não chegou a uma conclusão sobre os fatores que explicariam a associação entre volume e qualidade. Sugere-se que a experiência dos profissionais, a maior disponibilidade de recursos diagnósticos e terapêuticos, melhor organização e monitoramento dos processos de cuidados pré e pós-operatórios sejam mais prevalentes nos serviços de maior volume.

Eagle et al. (1999:1315), em extensa revisão bibliográfica para a produção de rotinas clínicas para CRVM, para a American Heart Association e American College of Cardiology, concluem na parte referente à associação entre volume e mortalidade: “os estudos sugerem quea 
sobrevida após CRVM é negati vamente afetada quando esta é real izada em institui ções que efetuam menos do que um número limite de casos anualmente... Esta observação fortal ece o argumento de que se monitorem instituições ou mé dicos que realizem menos de 100 cirurgias por ano. Deve ser lembrado que os estudos também revelaram uma ampla variação nas taxas de mortalidade ajustadas pelo risco em situações de baixo volume, isto é, algumas insti tui ções e médicos apresentam excelentes resultados a despei to de seus volumes rel ativamente baixos".

Concluindo, considera-se que apesar das limitações metodológicas deste estudo, o fato de seus resultados estarem em consonância com as evidências existentes de que hospitais com baixo volume de CRVM têm probabilidade de resultados desfavoráveis aumentada, permitem a recomendação de que no SUS deve-se estimular a concentração regionalizada dos serviços para a realização de CRVM. Sobretudo, se forem considerados também os custos de implantação e operação dos serviços hospitalares necessários para dar suporte a esses procedimentos. Problemas no acesso com conseqüente atraso no tratamento, que podem decorrer da concentração de CRVM em determinados hospitais, devem ser contrabalançadas com os ganhos decorrentes do aumento do volume de cirurgias. Por outro lado, ênfase deve ser dada ao monitoramento da qualidade do cuidado e a medidas de melhoria da qualidade, particularmente em áreas onde se faça necessária a contratação de serviços de menor volume. Nos Estados Unidos, organizações de planos privados de saúde começam a orientar seus profissionais de saúde e pacientes a selecionar serviços de maior volume e sugerem, no caso da CRVM, hospital com um mínimo de 500 cirurgias/ ano (Birkmeyer et al., 2002).

\section{Referências}

BIRKMEYER, J. D.; SIEWERS, A. E.; FINLAYSON, E. V.; STUKEL, T. A.; LUCAS, F. L.; BATISTA, I.; WELCH, H. G. \& WEN NBERG, D. E., 2002. Hospital volume and surgical mortality in the United States. New England Journal of Medicine, 346:1128-1137.

CAMPOS, M.; MARTINS, M.; NORONHA, J. C. \& TRAVASSOS, C., 2000. Proposta de integração de dados do Sistema de Informações Hospitalares do Sistema Único de Saúde (SIH-SUS) para pesquisa. Informe Epidemiológico do SUS, 9:51-58.

CLARK, R. E., 1996. Outcome as a function of annual coronary artery bypass graft volume. Annals of Thoracic Surgeons, 61:21-26.

DATASUS (Departamento de Informática do SUS), 1999. Movimento de Autorização de Internação Hospitalar: Arquivos Mensais. Períodos: janeiro 1996 a dezembro de 1998. CD-ROM. Brasília: Ministério da Saúde.

DONABEDIAN, A, 1980. The Definition of Quality and Approaches to its Assessment. Ann Arbor: Health Administration Press.

DUBOIS, R. W.; ROGERS, W. H.; MOXLEY III, J. H.; DRAPER, R. H. \& BROOK, R. H., 1987. Hospital inpatient mortality - Is it a predictor of quality. New England Journal of Medicine, 317:1674-1680. 
EAGLE, K. A.; GUYTON, R. A.; DAVIDOFF, R.; EWY, G. A.; FONGER, J.; GARDNER, T. J.; GOTT, J. P.; HERRMANN, H. C.; MARLOW, R. A.; NUGENT, W. C.; O'CONNOR, G. T.; ORSZULAK, T. A.; RIESELBACH, R. E.; WINTERS, W. L.; YUSUF, S.; GIBBONS, R. J.; ALPERT, J. S.; GARSON J r., A.; GREGORATOS, G.; RUSSELL, R. O. \& SMITH Jr., S. C., 1999. ACC/AHA guidelines for coronary artery bypass graft surgery: A report of the American College of Cardiology/ American Heart Association Task Force on Practice Guidelines. Journal of the American College of Cardiology, 34:1262-1347.

FINK, A.; YANO, E. M. \& BROOK, R. H., 1989. The condition of the literature on differences in hospital mortality. Medical Care, 27:315-336.

HANNAN, E. L.; KILBURN Jr., H.; BERNARD, H.; O'DONNELL, J. F.; LUKACIK, G. \& SHIELDS, E. P., 1991. Coronary artery bypass surgery: The relationship between inhospital mortality rate and surgical volume after controlling for clinical risk factors. Medical Care, 29:1094-1107.

HANNAN, R. G.; O'DONNELL, J. F.; KILBURN, H.; BERNARD, H. R. \& YAZICI, A., 1989. Investigation of the relationship between volume and mortality for surgical procedures performed in New York state hospitals. JAM A, 262:503-510.

JENCKS, S. F.; WILLIAMS, D. K. \& KAY, T. L., 1988. Assessing hospital associated deaths from discharge data: The role of length of stay and comorbidity. JAMA, 260:2240-2246.

KELLY, J. V. \& HELLINGER, F. J., 1987. Heart disease and hospital deaths: An empirical study. Health Services Research, 22:369-395.

LUFT, H. S.; GARNICK, D. W.; MARK, D. H. \& MCPHEE, S. J., 1990. Hospital Volume, Physician Volume, and Patient Outcomes: Assessing the Evidence. Ann Arbor: Health Administration Press.

LUFT, H. S.; HUNT, S. S. \& MAERKI, S. C., 1987. The volume-outcome relationship: Practice makes perfect or selective referral patterns? Health Services Research, 22:157-182.

MS (Ministério da Saúde), 2000. Manual da AlH. Rio de Janeiro: DATASUS.

NHS CENTRE FOR REVIEWS \& DISSEMINATION, 1997. Concentration and Choice in the Provision of Hospital Services. The Relationship Between Hospital Volume and Quality of Health Outcomes. CRD Report 8, Part I. York: The University of York.

NORONHA, J. C., 2001. Utilização de Indicadores de Resultados para a Avaliação da Qualidade em Hospitais de Agudos: Mortalidade Hospitalar após Cirurgia de Revascularização do Miocárdio em Hospitais Brasileiros. Tese de Doutorado, Rio de Janeiro: Instituto de Medicina Social, Universidade do Estado do Rio de Janeiro.
OTA (Office of Technology Assessment), 1988. Hospital mortality rates. In: Quality of Medical CareInformation for Consumers, OTA - H-386, Chapter 4, pp. 71-98, Washington, DC: Congress of the United States, Office of Technology Assessment.

PALMER, R. H., 1991. Considerations in defining quality of health care. In: Striving for Quality in Health Care (R. H. Palmer, A. Donabedian \& G. J. Povar, ed.), pp. 1-54, Ann Arbor: Health Administration Press.

ROTHMAN, K. \& GREENLAND, S., 1998. Modern Epidemiology. 2nd Ed. Philadelphia: LippincottRaven Publishers.

SHROYER, A. L.; MARSHALL, G.; WARNER, B. A.; JOHNSON, R. R.; GUO, W.; GROVER, F. L. \& HAMMERMEISTER, K. E., 1996. No continuous relationship between veterans' affairs hospital coronary artery bypass grafting surgical volume and operative mortality. Annals of Thoracic Surgeons, 61:17-20.

SOWDEN, A.; DEEKS, J. J. \& SHELDON, T. A., 1995. Volume and outcome in coronary artery bypass graft surgery: True association or artefact? BMJ, 311:151-155.

SPIEGELHALTER, D. J., 2002. M ortality and volume of cases in pediatric cardiac surgery: Retrospective study on routinely collected data. BMJ, 324:261264.

THOMAS, J. W. \& HOFER, T. P., 1998. Research evidence on the validity of risk-adjusted mortality rates as a measure of hospital quality of care. Medical Care Research and Review, 55:371-404.

TRAVASSOS, C.; NORONHA, J. \& MARTINS, M., 1999. Mortalidade hospitalar como indicador de qualidade - Uma revisão. Ciência \& Saúde Coletiva, 4:367-381.

TU, J. V. \& NAYLOR, C. D., 1996. Coronary artery bypass mortality rates in Ontario: A Canadian approach to quality assurance in cardiac surgery. Steering Committee of the Provincial Adult Care Network of Ontario. Circulation, 94:2429-2433.

TU, J. V.; SYKORA, K. \& NAYLOR, C. D., 1997. Assessing the outcomes of coronary artery bypass graft surgery: How many risk factors are enough. Journal of American College of Cardiology, 30:13171323.

WENNBERG, J. E.; ROOS, N. P.; SOLA, L.; SCHORI, A. \& JAFFE, R., 1987. U se of claims data systems to evaluate health care outcomes: Mortality and reoperation following prostatectomy. JAMA, 257: 933-936.

Recebido em 2 de outubro de 2002

Versão final reapresentada em 30 de abril de 2003

Aprovado em 11 de agosto de 2003 